\title{
Blue Variables from the MOA Database
}

\author{
Sachiyo Noda \\ Solar-Terrestrial Environment Laboratory, Nagoya University, Chikusa, \\ Nagoya 464-8601 Japan \\ Mine Takeuti \\ Astronomical Institute, Tohoku University, Aoba, Sendai 980-8578 \\ Japan
}
I. A. Bond ${ }^{1,4}$, N. J. Rattenbury ${ }^{1}$, F. Abe ${ }^{2}$, B. S. Carter ${ }^{3}$, R. J. Dodd ${ }^{3}$, J. B. Hearnshaw ${ }^{4}$, M. Honda ${ }^{5}$, J. Jugaku $^{6}$, S. Kabe $^{7}$, P. M. Kilmartin ${ }^{1,4}$, K. Masuda ${ }^{2}$, Y. Matsubara ${ }^{2}$, Y. Muraki ${ }^{2}$, T. Nakamura ${ }^{8}$, M. Reid ${ }^{9}$, N. J. Rumsey ${ }^{3}$, To. Saito ${ }^{10}$, H. Sato ${ }^{8}$, M. Sekiguchi ${ }^{5}$, D. J. Sullivan ${ }^{9}$, T. Sumi ${ }^{2}$, Y. Watase ${ }^{7}$, T. Yanagisawa ${ }^{2}$, P. C. M. Yock ${ }^{1}$, M. Yoshizawa ${ }^{11}$

\begin{abstract}
A large database of CCD photometry for 1.4 million stars towards both the LMC and the SMC, which has been established by the MOA project, is a useful resource to study variable stars. In our preliminary study, variables identified as $\beta$ Lyrae type stars and Herbig $\mathrm{Ae} / \mathrm{Be}$ stars have been found amongst blue stars.
\end{abstract}

The MOA database is a product of large-scale CCD photometry searching for gravitational microlensing events which are possibly caused by MACHOs in the galactic halo. Details are described in Abe et al. (1997), Muraki et al. (1999), and also by Hearnshaw et al. (2000).

The original aim was to search for microlensing events, and for this purpose the observational data obtained from 1997 January to 1998 July were analysed with the following steps:

\footnotetext{
${ }^{1}$ University of Auckland, Auckland, New Zealand

${ }^{2}$ Solar-Terrestrial Environment Laboratory, Nagoya University, Chikusa, Nagoya, 464-85601, Japan

${ }^{3}$ Carter National Observatory, Wellington, New Zealand

${ }^{4}$ Department of Physics and Astronomy, University of Canterbury, Canterbury, New Zealand

${ }^{5}$ Institute for Cosmic Ray Research, University of Tokyo, Tanashi 188, Japan

${ }^{6}$ Institute for Civilization, Sakuragaoka 2-29-3, Tama 206, Japan

${ }^{7}$ National Laboratory of High Energy Physics (KEK), Tsukuba 305, Japan

${ }^{8}$ Department of Physics, Kyoto University, Kyoto 606, Japan

${ }^{9}$ Department of Physics, Victoria University, Wellington, New Zealand

${ }^{10}$ Tokyo Metropolitan College of Aeronautics, Tokyo 140-0011, Japan

${ }^{11}$ National Astronomical Observatory, Mitaka, Tokyo 181-8565, Japan
} 
1. selection of the stars whose number of observed nights exceeds 100 for the LMC members, and 80 for SMC members (327647 stars in the LMC, and 146358 stars in the SMC);

2. selection of stars with a large deviation in radiative flux from the random fluctuation, the Gaussian distribution, by checking their $\chi^{2}$ values. (In the LMC we chose 43801 stars with the condition $\chi^{2}<4.3$, and in the SMC 18018 stars with another condition, $\chi^{2}<3.3$ );

3. selection of stars with small systematic changes in the colour compared with the variation in brightness to find microlensing events with a relative threshold of $\chi_{\text {colour }}^{2} \leq 0.2 \chi_{\text {blue }}^{2}$ or $0.2 \chi_{\text {red }}^{2}$ (22776 and 6114 stars remained in the LMC and the SMC);

4. selection of stars whose amplitude of colour variation is smaller than a criterion, $\sigma_{\text {colour }}^{2} \leq 0.003$ for the LMC and 0.002 for the SMC.

A total of 485 stars in the LMC and 305 stars in the SMC remained after these selections had been carefully performed.

We checked all the star in these remaining 790 datasets. No candidate microlensing events have been found. To study the nature of light variation in each star, we first tried to find a periodicity. After finding the period, we classified each star by plotting the light curve of phased data and comparing the features of the light curve and the position of the star in the colour-magnitude diagram.

Table 1. Blue variable stars selected after step 4 (numbers including red variables are shown in parenthesis)

\begin{tabular}{lcccc}
\hline & \multicolumn{2}{c}{ LMC } & \multicolumn{2}{c}{ SMC } \\
\hline Eclipsing binaries & 64 & $(69)$ & 132 & $(136)$ \\
Cepheids & 6 & $(78)$ & 21 & $(38)$ \\
Semiregular variables & 0 & $(188)$ & 0 & $(35)$ \\
Others and unclassified & 76 & $(150)$ & 90 & $(96)$ \\
Total & 146 & $(485)$ & 243 & $(305)$ \\
\hline
\end{tabular}

The numbers of variables whose colour satisfies a condition $\left(B_{\mathrm{MOA}}-R_{\mathrm{MOA}}\right)<0$ amongst the stars selected after step 4 are tabulated. Eclipsing binaries with $\beta$ Lyrae-type features and irregular variables presumed to be Herbig $\mathrm{Ae} / \mathrm{Be}$ stars were found.

\section{References}

Abe, F., Allen, W., Banks, T., et al. (The MOA Group) 1997, in Variable Stars and the Astrophysical Returns of the Microlensing Surveys, ed. R. Ferlet, J.-P. Maillard, \& B. Raban (Gif-sur-Yvette: Editions Frontières), 75

Hearnshaw, J. B., Bond, I. A., Rattenbury, N. J., et al. (The MOA Group) 2000, IAU Coll. 176, in these proceedings, p. 31

Muraki, Y., Sumi, T., Abe, F., et al. (The MOA Group) 1999, Prog. Theor. Phys. Suppl., No. 133, 233 